\title{
A Clinical Study on sources of Catheter Based Urinary Tract Infections
}

\author{
Karan Vats, Manjit Tanwar and Shalinder Koul \\ Department of General Surgery Shree Guru Gobind Singh \\ Tricentary University, Gurugram, Haryana, India \\ Corresponding author email: karanvats_fmhs@sgtuniversity.org
}

\section{ABSTRACT}

Hospital acquired infections specifically urinary tract infections have been commonly observed in developing countries. Urinary tract infections account for one fourth of hospital acquired infections. The patients' exposure to pathogens has been mostly linked with their stay in critical units of health care centers. The other most common cause of these infections has been compromised immune system of patients. The present study focuses on evaluating other factors that can cause UTIs. The results show prevalence of four bacterial species - S. aureus, P. aeriginosa, K. pneumoniae and E. fecalis in clinical samples of urinary catheter, urine bags, patients' blood and urine specimens. The study also evaluates role of catheter maintenance in causing UTIs and it has been found that hygienic practices such as cleaning of urine bag at proper time and disinfection of catheter after urination can help in controlling UTIs. This study provides a perspective of finding unconventional sources responsible for UTI which can help in prevention of catheter based infections in future.

KEY WORDS: CATHETER MAINTENANCE, NOSOCOMIAL INFECTION, URINARY CATHETER, URINARY TRACT INFECTIONS (UTIS), URINARY BAG

\section{INTRODUCTION}

Nosocomial infections or Hospital acquired infections are the infections which are caused due to patients' exposure to pathogens when they visit health care centers or hospitals. The prevention of these infections has developed prominence and they have emerged out in spotlight by research studies. The research initiatives have been directed towards wound infections, blood infections and pneumonia developing from ventilators. The another aspect of urinary tract infections remained underestimated, which is a threat as urinary infections are most common infections acquired from hospitals. The prevalence rate of UTIs (Urinary tract infections) accounts for $32.2 \%$ of all hospital acquired infections (S. M. Zahraei et. al. (2012)). Around $80 \%$ of

Biosc Biotech Res Comm P-ISSN: 0974-6455 E-ISSN: 2321-4007

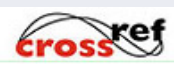

Identifiers and Pagination

Year: 2021 Vol: 14 No (6) Special Issue

Pages: 435-439

This is an open access article under Creative

Commons License Attribn 4.0 Intl (CC-BY).

DOI: http://dx.doi.org/10.21786/bbrc/14.7.91 these UTIs are cause due to urinary catheters and mostly affects patients who are acutely ill. UTIs develop bacterial infection in blood of $2-4 \%$ patients with mortality rate of around 13\% (J. M. T. Brusch et. al. (2020).

A urinary catheter is an invasive tube placed in the urinary organs for drainage and collection of urine from the bladder. The catheters are prescribed to patients when they suffer with uncontrollable urination, problems in urinary retention and drainage, during prostate surgery or when patients suffer from complex diseases such as multiple sclerosis, spine injury or dementia. Based on requirements and patients' convenience related to their body disorders, catheters can be of various types (Table1) and materials (latex, silicon, Teflon) (Urinary catheters et. al. (2020)). Foley catheter is most commonly used as rubber tube inserted in the patients' bladder for draining out the urine(S. Garg et. al. (2010)). The patients' susceptibility to develop UTI through indwelling catheter is based on several factors such as age, sex, duration of hospital stay, severity of disease requiring hospital stay, antimicrobial therapy, duration of using the catheter and catheter maintenance practices.
Article Information

Received: $18^{\text {th }}$ May 2021

ccepted after revision: $30^{\text {th }}$ July 2021 
Table 1. Different types of urinary catheters used by male and female patients(https://www.urotoday.com/ urinary-catheters-home/indwelling-catheters/description/definitions.html, https://www.shopcatheters. com/p-rusch-easycath-female-intermittent-catheter.html)

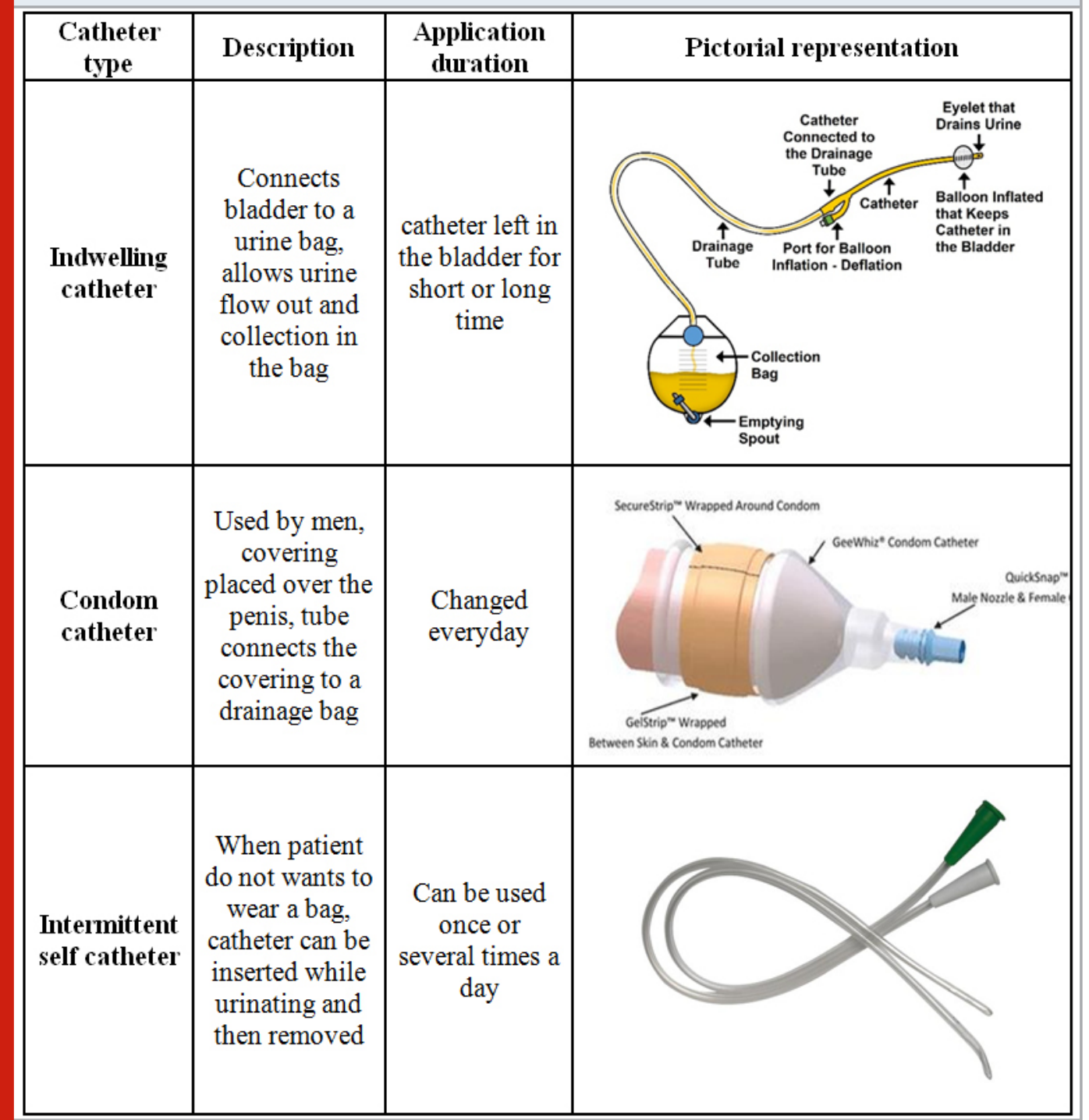

Literature Review: Urinary tract infections has affected the course of disease progression and promoted the risk of other diseases. UTI can cause kidney inflammation due to bacterial infection progression to kidney, premature delivery and fetal death in pregnant women. The infection affects renal function and cause end stage renal disease in patients suffering from pediatrics. According to studies based on catheter associated UITs, the prevention strategies have been mostly based on population of intensive care unit (ICU). It has been observed that out of total 506 UTIs acquired from catheters use, $72 \%$ were found in non-ICU patients and $28 \%$ were found in ICU patients. The catheter associated bacterial infection was found in $9.6 \%$ cases (B. Fozman et. al. (2003)). Overall it can be concluded that prevalence of UTIs cannot be associated with ICUs in general. The infection may be based on other parameters and health care practices.
It has already been reported that most of the hospital acquired infections, including urinary tract infections are directed towards immune compromised patients. Due to this, several studies have been conducted on ICUs as the room sustains most of the critically ill patients. However, several other studies have observed UTIs as hospital acquired infections in other patients as well. Although, the susceptibility of immuno-compromised patients is technically logical but the results cannot over-mask the possibility of other reasons that can increase the probability of UTIs in hospitals. With the upcoming time the complexity of infections has elevated to numerous folds. The UTI patients treated with antimicrobials have started attaining resistance towards common therapies due to evolution of bacterial defense. The infections have taken a form of resistant biofilm usually develop inside the catheters, deteriorating patients health conditions (H. Pelling et. al. (2019)). Therefore, the present study is based on finding other sources of UTIs through 
microbiological analysis. The study further discussed the susceptibility of immuno-compromised patients and antimicrobial resistance in UTIs.

\section{Research Questions}

1. What are the bacterial species present in clinical samples of catheters?

2. What could be other possible cause of UTI - urine bag, catheter maintenance, catheter biomaterial?

\section{MATERIAL AND METHODS}

Sample collection: To conduct this study, Foley catheters were used as samples for studying microbial prevalence in urinary catheters along with specimens of urine drainage bag, patient's blood and urine. A foley catheter is an indwelling catheter mostly used for draining of urine from bladder to a urine bag. The catheter is made of soft material usually rubber or plastic to provide flexibility during application and convenience to users. In this study, used foley catheters were collected from regional hospital in Delhi and categorized based on different materials namely - Teflon, Latex and Silicon. The catheter owner information was collected through patient information form (PIF) including questionnaireregarding patient's demographics and disease history. The form also contains a section on catheter maintenance practices developed by the user for cleaning of catheter (Table 2).

Table 2. Patient information form used in the study.

\begin{tabular}{|c|c|c|}
\hline Category & Questions & Response \\
\hline \multirow{4}{*}{ Demographics } & Patient ID & (specify) \\
\hline & Age & $\begin{array}{l}\text { Below } 30,30-50,51-70,70 \\
\text { and beyond }\end{array}$ \\
\hline & Duration of stay & $\begin{array}{l}\text { Below } 2 \text { weeks, } 2-4 \text { weeks, } \\
\text { more than } 4 \text { weeks }\end{array}$ \\
\hline & Disease history & (specify) \\
\hline \multirow{7}{*}{$\begin{array}{l}\text { Catheter } \\
\text { maintenance }\end{array}$} & How often do you change the catheter? & 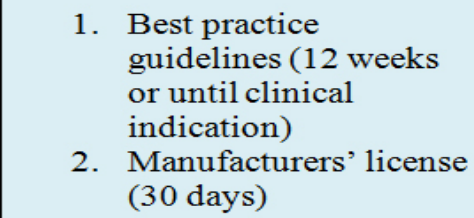 \\
\hline & What do you use for washing the catheter? & $\begin{array}{l}\text { Only Water, regular Soap, } \\
\text { Antimicrobial detergent }\end{array}$ \\
\hline & $\begin{array}{l}\text { Do you use hand gloves while changing the } \\
\text { catheter? }\end{array}$ & Yes / No \\
\hline & How often the urine bag is changed? & $\begin{array}{l}\text { Every } 6 \text { hours, every } 24 \text { hours, } \\
\text { When full }\end{array}$ \\
\hline & Any experience of abdominal pain? & Yes / No \\
\hline & Have you submitted your urine sample? & Yes / No \\
\hline & Have you submitted your blood sample? & Yes / No \\
\hline
\end{tabular}

Assessment of Patient's health: The bacterial presence in the catheter was studied by in vitro culturing methods. The used catheters were washed by phosphate buffer saline (PBS) once and then placed in a test tube filled with sterile Luria Bertani media (LB) as experimental setup. The test tubes were incubated at 37o C, $250 \mathrm{rpm}$ ( 24 hours). The turbidity observed in media was quantified by measuring optical density at $260 \mathrm{~nm}$ and Colony forming units were evaluated. Similarly, blood and urine samples were also cultured by serial dilution method to study the progression of the infection. Specimens of urine drainage bags were also cultured to track the source of infection. Additionally, immunoglobulin test was conducted using patients' blood samples to study immune deficiency ad its relation with catheter infections.

Identification of bacterial species: The identification of bacterial species in different in-vitro sample cultures was done by demonic DNA isolation of different colonies observed after culture plating. The isolated DNA was amplified by 16 S rRNA PCR (Polymerase Chain Reaction) using ThermofischerMasterCycler. The obtained genetic samples were sent for sequencing to commercial service provider. The results were obtained through email and sequence similarity search was performed using Basic Local Alignment Search Tool. The results obtained were sorted based on inclusion parameters as more than $80 \%$ sequence homology. The sequence database was used to identify the bacterial species most closely related to the sequencing results.

Data analysis and statistics: The data obtained through patient information form was statistically analyzed through Origin Pro software and one way ANOVA was used to determine statistical significance. The graphical representation of data was done on Microsoft excel. The 
data obtained through patients blood and urine report was simplified in the form of graphical arrangement to provide reader convenience and understandable to patients also.

\section{RESULTS AND DISCUSSION}

Bacterial prevalence on catheter surface: To determine the susceptibility of catheter material towards adhesion of bacterial pathogens was determined by in vitro culturing of used catheters of materials Teflon, latex and silicon. The unused catheter was considered as negative control. As shown in Figure 1, it was observed that maxiumum umber of colony forming units per square centimeter of catheter were obtained from samples of latex catheters, followed by teflon catheters. The difference in CFU of Teflon and latex was less which may be due to mixing of some Teflon in latex catheters also. Thus, a clear distinction could not be made between the two. However, silicon catheter showed least amount of bacterial population. Thus, it can be said that silicon catheters might be better choice as compared to other catheter materials to prevent bacterial infections. However, the adhesion would be dependent on catheter surface characteristics and type of pathogen species.

Figure 1: Bacterial growth over catheters of different biomaterial

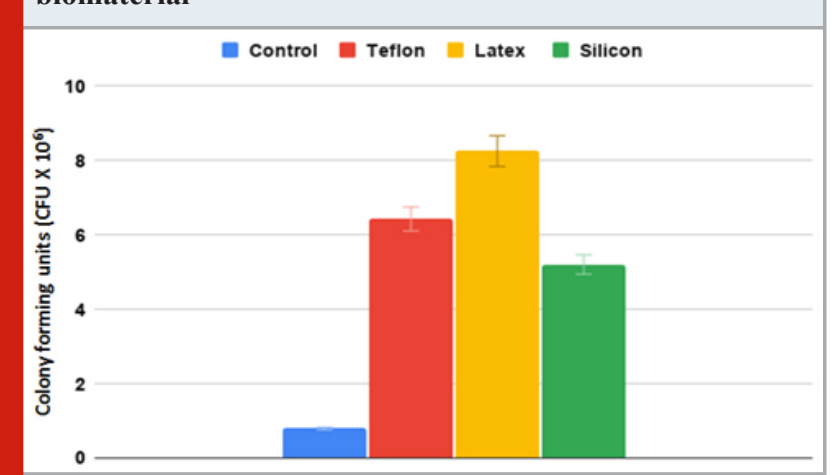

Figure 2: Collective prevalence of bacterial species in catheters, urine bags and blood specimens of patients.

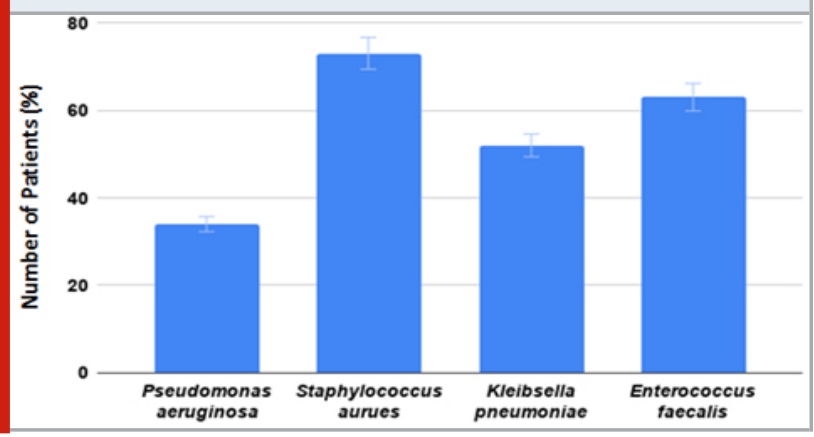

Bacterial species prevalence in patients: In order to identify bacterial species prevailing in catheters, species identification was done on samples of foley catheters, urine bag and blood samples. The collective data was presented based on different species identified from the samples. According to Figure 2, it was found that S. aureus was the most prevalent bacteria species (more than $70 \%$ ) as compared to P. aeriginosa, K. pneumoniae and E. fecaliswhich were identified to be present in samples of catheters, urine bags as well as patients' blood. Another most prevalent species is E. faecaliswhichhas been mostly associated with bacterimic conditions arising from catheter infections. Moreover, if only blood samples were analysed, E. faecalisis the most prevalent species (data not shown) depicting the bacterimic caused to be associated with catheters. Further most of these species, specifically S. aureus, P. aeruginosa and E. faecalis have been known for forming bacterial biofilms over medical devices(S. J. Cole (2014), J. H. Ch'ng (2019)). The presence of these species creates threat for serious infections which may also develop resistance to antimicrobial therapies(R. Boss et. al. (2016)). The study could be further explored to test the presence of resistant bacteria species by performing antibacterial screening in future.

Figure 3: a) Presence of Urinary tract infections (UTIs) according to hospital stay duration of a patient. b) Immune system analysis based on hospital stay duration of a patient.

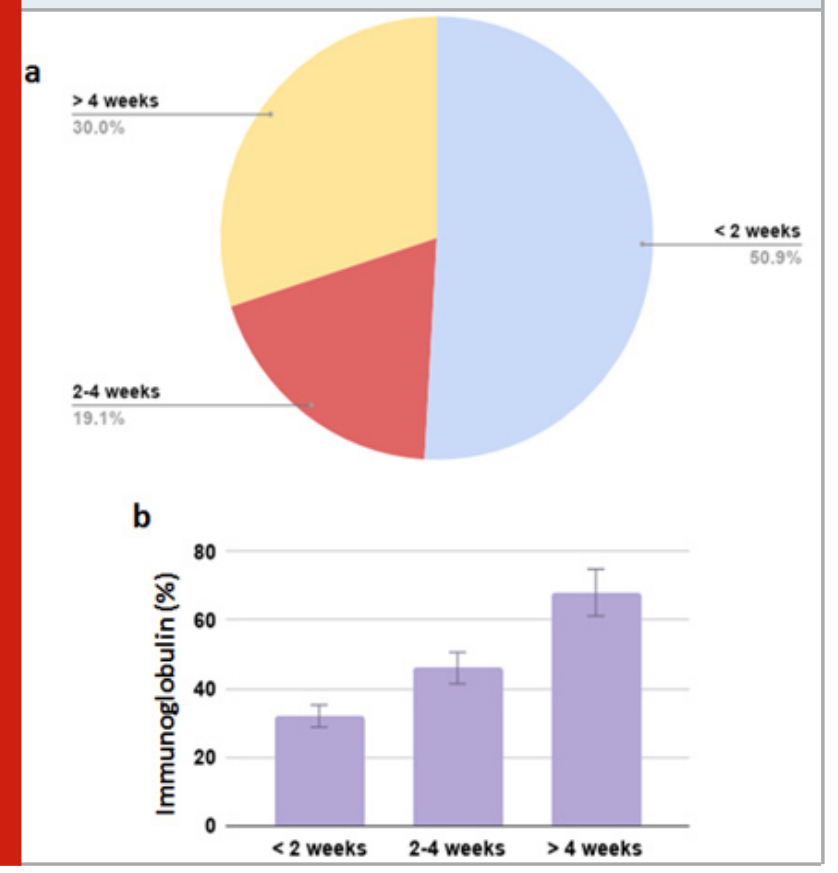

Relation between UTIs and hospital stay: The presence of threatening bacterial species among specimens collected from patients admitted in the hospital provides a hint of hospital settings as possible cause of infection. To estimate the correlation, the responses obtained in patient information form were analyzed. The stay was divided into options as less than 2 weeks, 2-4 weeks and more than 4 weeks. It was observed that most of the UTI positive cases were found to be staying in hospital for less than 2 weeks. Less number of patients who stayed for 4 or more weeks showed UTI positive results. Further, the immune system was also analyzed for susceptibility to cause UTIs. It was found that patients staying for less than 2 weeks had weakest immune system. Thus, it can be said that patients having compromised immune system can be more susceptible to have UTI. Overall, t results suggest that UTIs may or may not have nosocomial origin but UTI have high chances to 
infect a person whose immune system is relatively weaker. There could be other sources of infections as well, which can also be related to the maintenance practices that the patient performs while using the catheter (Figure 3).

Catheter maintenance and UTI: The results obtained till now indicate that there could be other sources of infections possible apart from nosocomial origin. These catheters are sometimes used by patients on their own in routine life. The handling of catheters without expert supervision can lead to frugal ways of handling the catheter which can give rise to bacterial infections. One of the major entry pathways for UTI could be inappropriate handling of catheters and urine bag associated with it. Hence, catheter maintenance questions were asked from the patients to gain an idea of catheter handling practices leading to UTIs. According to Figure $4,70 \%$ of UTI positive cases change their urine bags when they are full. This practice could create unhygienic conditions in the bag due to fouling of urine. The fouling pathogens can travel towards bladder causing UTIs and other severe diseases. Moreover, people who change their bags at shorter intervals showed less positive cases for UTIs.

Figure 4: Catheter maintenance practices that may cause urinary tract infections (UTIs)

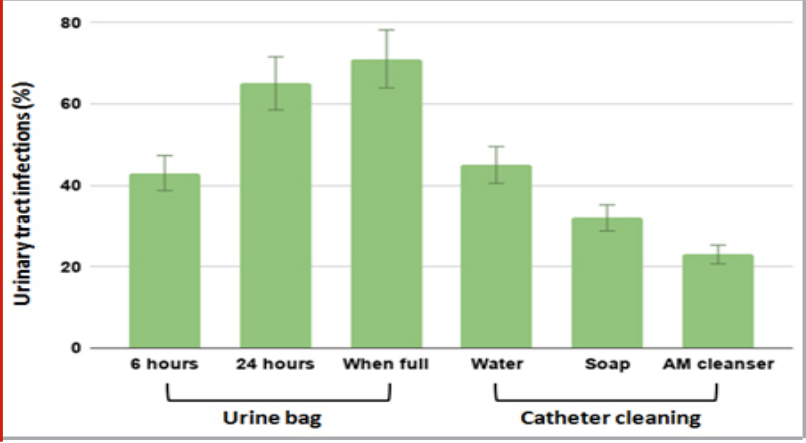

The entry of pathogens while putting up a catheter can also cause bacterial infections. It was asked if patients wear gloves while changing the catheter. It was found from the responses that very few people wear gloves while changing or washing the catheters after urination. It was found that most UTI positive cases use only water rinse to wash the catheters. Also, people using antibacterial washing agent have been found less positive for UTIs. Therefore, it can be concluded that washing and maintenance of catheters while being handled by patients can prevent bacterial infections in urinary organs. Further, catheter maintenance practices of doctors and experts can be also studied in future to determine the source of UTIs.

\section{CONCLUSION}

The increasing incidences of urinary tract infections and elevated mortality rate due to successive ailments have attracted researchers' attention towards identifying the cause of UTIs. These infections have been associated with nosocomial origin and immune system of the patients. It has been reported that patients with compromised immune system are more susceptible to UTIs. Contradicting studies exist regarding the sources of pathogens responsible for UTIs creating ambiguity of exact cause of infections. The present study has evaluated prevalence of bacterial species on used catheter samples obtained from hospitals. Further, it has been found that four bacterial species - S. aureus,P. aeriginosa, K. pneumoniae and E. fecaliswere prevalent in clinical samples of catheters, urine bags and blood specimens of patients dependent on catheters. This study also evaluated catheter maintenance practices that may lead to UTIs. Overall, the study presents practical data based on possible causes of UTIs based on urinary catheters.

\section{REFERENCES}

Bhatia, N., Daga, M.K., Garg, S. and Prakash, S.K., 2010. Urinary catheterization in medical wards. Journal of global infectious diseases, 2(2), p.83.

Boss, R., Overesch, G. and Baumgartner, A., 2016. Antimicrobial resistance of Escherichia coli, Enterococci, Pseudomonas aeruginosa, and Staphylococcus aureus from raw fish and seafood imported into Switzerland. Journal of food protection, 79(7), pp.1240-1246.

Ch'ng, J.H., Chong, K.K., Lam, L.N., Wong, J.J. and Kline, K.A., 2019. Biofilm-associated infection by enterococci. Nature Reviews Microbiology, 17(2), pp.82-94.

Cole, S.J., Records, A.R., Orr, M.W., Linden, S.B. and Lee, V.T., 2014. Catheter-associated urinary tract infection by Pseudomonas aeruginosa is mediated by exopolysaccharide-independent biofilms. Infection and immunity, 82(5), pp.2048-2058.

Foxman, B., 2003. Epidemiology of urinary tract infections: incidence, morbidity, and economic costs. Disease-a-month, 49(2), pp.53-70.

Mohsin, J., Weerakoon, S., Ahmed, S., Puts, Y., Al Balushi, Z., Meis, J.F. and Al-Hatmi, A., 2020. A cluster of Candida auris blood stream infections in a tertiary care hospital in Oman from 2016 to 2019. Antibiotics, 9(10), p.638.

Pelling, H., Nzakizwanayo, J., Milo, S., Denham, E.L., MacFarlane, W.M., Bock, L.J., Sutton, J.M. and Jones, B.V., 2019. Bacterial biofilm formation on indwelling urethral catheters. Letters in applied microbiology, 68(4), pp.277-293.

Waldrop, M. and Horton, L., 2020. NURS 360: PICO Question: In males, do external urinary catheters or indwelling urinary catheters provide better outcomes for the patient?.

Zahraei, S.M., Eshrati, B., Asl, H.M. and Pezeshki, Z., 2012. Epidemiology of four main nosocomial infections in Iran during March 2007-March 2008 based on the findings of a routine surveillance system. Archives of Iranian medicine, 15(12), pp.0-0. 